Devenir moderne, rester Chinois : Pratiques discursives autour de l'Institut franco-chinois de Lyon (1921-1946)

Florent VILLARD

\title{
OpenEdition
}

Journals

Édition électronique

URL : http://journals.openedition.org/transtexts/570

DOI : $10.4000 /$ transtexts. 570

ISSN : 2105-2549

Éditeur

Gregory B. Lee

Référence électronique

Florent VILLARD, «Devenir moderne, rester Chinois : Pratiques discursives autour de l'Institut francochinois de Lyon (1921-1946) », Transtext(e)s Transcultures 跨文本跨文化 [En ligne], 9 | 2014, mis en ligne le 13 novembre 2015, consulté le 19 avril 2019. URL : http://journals.openedition.org/ transtexts/570 ; DOI : 10.4000/transtexts.570

Ce document a été généré automatiquement le 19 avril 2019.

(c) Tous droits réservés 


\title{
Devenir moderne, rester Chinois : Pratiques discursives autour de l'Institut franco-chinois de Lyon (1921-1946)
}

\author{
Florent VILLARD
}

1 Lorsque, dans les années mille neuf cent vingt, les personnalités prestigieuses que sont devenus Zhang Xi 张永, Li Heng 李行, Luo Dagang 罗大刚, Dai Wangshu 戴望舒, Zhang Ruoming 张若名, Zheng Yanfen 郑彦蔡, Pan Yuliang 潘玉良 ou Su Xuelin 苏雪林 ( futures astronomes, physiciens, politiciens, poètes, peintres) s'embarquaient depuis la Chine sur un navire des Messageries maritimes à destination de Marseille, ils effectuaient un déplacement géographique, social et culturel pour s'installer, pendant quelques années, dans les locaux de l'Institut franco-chinois au Fort St Irénée de Lyon. Entre 1921 et 1946, 473 étudiants chinois, dont 51 femmes, originaires de différentes provinces, deviendront pensionnaires de l'Institut. Environ 140 d'entre eux soutiendront une thèse de doctorat dans le cadre du système universitaire français, pour la plupart à l'Université de Lyon. Les disciplines étudiées par ces étudiants couvraient l'ensemble du champ académique de l'époque: Médecine, Sciences naturelles, Biologie, Pharmacie, Mathématiques, Lettres, Géographie, Histoire, Droit.

2 Les intellectuels chinois à l'initiative de cette entreprise projetaient sur cette expérience le mythe d'un métissage sino-français harmonieux et équilibré, susceptible d'engendrer une civilisation supérieure : «La création de cette université permettrait d'établir le lien entre les civilisations orientales et occidentales, de faire fusionner les sciences chinoises et étrangères, de créer une sorte de 'nouvelle civilisation', et d'inaugurer une ère nouvelle pour le genre humain. $»^{1}$ Li Shizeng, co-fondateur de l'IFCL, évoquera même «la formation d'une race franco-chinoise $»{ }^{2}$

3 Cet imaginaire syncrétique orientalisant était-il vraiment autre chose qu'un discours convenu pour sauver la face de la position chinoise dans cette collaboration ? Le mythe de la fusion ne tient pas compte du déséquilibre des forces en présence et de l'hégémonie 
culturelle et intellectuelle de la France, et de l'Europe, sur la Chine. ${ }^{3}$ Que cette perspective idéaliste des acteurs chinois fut soutenue par une conception universaliste et déterministe $d u$ progrès humain héritée des Lumières françaises confirme cette domination épistémologique de l' " Occident » sur la Chine.

En contradiction apparente avec la vision neutre et a-politique du métissage, le discours des acteurs de l'époque assurait aussi que les étudiants, en se rendant en Europe, effectuaient un déplacement qualitatif dans le temps : du passé vers le futur, de la tradition vers la modernité. Cette représentation du monde et de l'histoire à partir de l'idée d'un temps linéaire universelle était devenue dominante en Europe au XIX ${ }^{\mathrm{e}}$ siècle. ${ }^{4}$ Tandis que les sociétés à l'origine de ce discours se situaient en haut de l'échelle (Europe, $\mathrm{EU})$, le reste du monde devait s'efforcer de refaire péniblement son retard, avec l'aide des pays les plus avancés: En France, on parlait de "mission civilisatrice», terme indissociable de l'épopée coloniale.

5 Initialement centrés sur les thèses de doctorat des étudiants, nos travaux sur l'Institut franco-chinois de Lyon se sont progressivement ouverts à d'autres types de textes et de documents d'archives. ${ }^{5}$ La correspondance et les dossiers scolaires de certains étudiants, les Annales franco-chinoises éditées par l'Institut, les rapports du Conseil d'administration et une partie des archives administratives sont venus compléter les témoignages d'anciens étudiants, les articles de la presse locale et quelques précieuses photographies sur la vie quotidienne à l'Institut. ${ }^{6}$ La consultation de ces documents offrait une vision plus cohérente de cette expérience et permettait désormais d'envisager dans sa totalité cet espace géographique, social, intellectuel et culturel.

Dans le prolongement de nos travaux sur l'affirmation d'une subjectivité chinoise dans les paratextes des productions scientifiques des étudiants, cet essai se propose d'interpréter l'expérience de l'IFCL à travers la notion foucaldienne de dispositif. Foucault propose ce concept pour définir un ensemble de pratiques, de discours et d'institutions qui, dans une configuration historique donnée, participent de la fabrication de sujets sociaux déterminés.7 Cet assujettissement au pouvoir social n'empêche pas des formes d'appropriations par les sujets eux-mêmes - de subjectivation dira Foucault -du rôle auquel ils sont assignés : « Le sujet n'est pas 'naturel', il est modelé à chaque époque par le dispositif et les discours du moment, par les réactions de sa liberté et par ses éventuelles 'esthétisations' ».8

7 Si l'expérience particulière de l'IFCL n'épuise pas le processus, nous faisons l'hypothèse qu'elle cristallise néanmoins des pratiques discursives qui ont contribué à façonner un "sujet chinois moderne», expression que le présent essai devra tenter d'éclairer. Une définition simple et tautologique dirait que le «sujet chinois moderne» désigne des étudiants qui, à l'époque, s'identifient, et sont identifiés, simultanément en tant que «Chinois » et en tant que moderne. A première vue, l'hypothèse apparait bien peu audacieuse tant elle correspond aux objectifs de cette institution éducative. L'environnement scientifique et culturelle de cette école de préparation aux études supérieures en France avait pour finalité principale de former cette diaspora intellectuelle temporaire afin qu'elle joue ensuite son rôle historique dans le devenir moderne de la Chine. Le label d'école de la modernité est donc plus que légitime. Par contre, affirmer que l'Institut puisse avoir été un lieu où des individus se sont constitués en tant que « sujets chinois » constitue une proposition moins évidente. sentiment d'appartenance chez les étudiants, est sous-tendue par une interprétation 
constructiviste et moderniste de la nation, communauté politique qui n'est pas antérieure aux discours nationalistes et aux dispositifs institutionnels qui l'ont accompagné. ${ }^{9} \mathrm{En}$ Chine, l'imaginaire instituant qui permet l'émergence balbutiante d'une conscience nationale à l'aube du $\mathrm{XX}^{\mathrm{e}}$ siècle doit se comprendre dans le cadre d'une "mise en contexte mondiale » de la société chinoise. ${ }^{10}$ L'affirmation particulariste - soutenue par les critères de la race, de l'ethnie, de la langue ou de la culture - a été constitutive et simultanée avec la prise de conscience d'une certaine globalité, condition de la modernité. ${ }^{11}$ Les identités culturelles et nationales de la périphérie se sont construites dans un jeu de contraste avec l'« Occident » en tant que figure normative centrale de la modernité. ${ }^{12}$ Elles étaient, par défaut, ce que n'était pas le moderne occidental. Cette concomitance entre l'intégration de la Chine dans une modernité capitaliste globale et le développement d'un imaginaire national se trouve parfaitement illustrée par l'expérience de l'IFLC, synecdoque utopique d'une Chine revêtue simultanément des habits (neufs) de la « sinité » et de la modernité.

Dans cet espace où les étudiants s'appliquent à devenir des hommes de lettres et de science modelés par les institutions, les méthodes, les valeurs et les épistémologies du champ académique français, dans ce lieu où les étudiants découvrent les sports et les loisirs «modernes » que sont le football, le tennis, le cinéma, le billards, etc., entre ces murs où les étudiants doivent apprendre à se comporter en hommes/femmes de la civilisation moderne occidentale, la Chine en tant que référent identitaire est, par contraste, omniprésente.

10 Les discours culturalistes exaltant les particularismes sont souvent plus vigoureux dans les communautés d'exilés que sont les diasporas qu'au sein même du pays natal. A Lyon, l'éloignement géographique, l'isolement et l'environnement radicalement autre ont pu contribuer à l'exacerbation d'un imaginaire national. De même, le contraste entre ces étudiants - le monde d'où ils venaient - et le contexte français, se trouvait validé par les représentations et discours de la presse locale française, mais aussi, dans un autre registre, par ceux des milieux politiques et universitaires qui, sans cesse, réaffirmaient le brevet de " sinité » de ces étudiants. Le rôle auquel dirigeants français et chinois avaient assignés ces étudiants - devenir l'élite technique et scientifique de la Chine afin de participer à sa modernisation - renforçait d'autant ces processus de subjectivation: «Chinois» par essence, les étudiants devenaient aussi «Chinois» par conviction: des patriotes.

11 Les archives accréditent l'idée d'un espace singulier où a pu se déployer un imaginaire et des pratiques participant à l'affirmation d'une culture nationale. Les initiateurs du projet ont toujours eu le souci de cultiver la sinité des étudiants à travers l'apprentissage du mandarin, la valorisation des arts de la Chine (expositions de peinture, concerts de musique) et la constitution d'une bibliothèque sinologique incluant un fonds conséquent de textes et d'œuvres de la Chine pré-moderne. ${ }^{13}$ Chaque année, en octobre, le rituel patriotique - tradition moderne inventée au début des années 1910s - de la fête nationale donnait lieu à des cérémonies importantes. La diversité des origines géographiques et des positions politiques était réelle parmi les étudiants. Mais les dirigeants de l'Institut avaient le souci de l'unité - masquée comme une neutralité - idéologique et politique des étudiants, et se méfiaient des tendances communistes et fédéralistes. Le discours métapolitique du salut de la patrie par la science, finalité ultime de l'Institut, s'imposait aux étudiants. De même, si les pratiques culturelles et linguistiques apparaissent initialement diverses, les témoignages révèlent des processus d'homogénéisation. La 
communauté de destin se prolongeait jusque dans la mort avec la réalisation, en 1928, d'un monument dédié aux étudiants décédés dans le cimetière de Loyasse de Lyon. ${ }^{14}$

\section{Généalogie coloniale de l'IFCL}

Dans un article où il narre l'histoire de l'Association universitaire franco-chinoise, créée en juillet 1921 et qui va servir de structure légale pour l'Institut (elle sera contrôlée par les français), l'orientaliste Maurice Courant, premier professeur de chinois de l'Université de Lyon, cheville ouvrière dans la création de l'Institut et secrétaire général de l'Association entre 1921 et 1934, nous livre une généalogie édifiante de l'IFCL :

Le fil de soie qui figure sur les médailles de la Chambre de Commerce de Lyon, bien que ténu, relie solidement notre ville à la Chine. Dans la première mission officielle envoyée par le Gouvernement Français au Gouvernement Chinois, dans la Mission Lagrené, nous trouvons des lyonnais de voisinage ou d'adoption, Isidore Jedde, Natalis rondot que beaucoup d'entre nous ont connu : ce sont-là les pionniers d'il y a 80 ans. Bien plus tard, quand la France à pu de nouveau s'intéresser aux relations lointaines, Paul Brunat a marqué la voie vers les affaires de Chine ; et, peu après, Ulysse Pila, dont le souvenir est encore si vivant, a été l'un des animateurs de cette mission d'études commerciales, 1895-1897, qui a fourni tant de renseignements et a laissé tant de traces. ${ }^{15}$

L'histoire culturelle, qui est une histoire des représentations, se penche sur le discours des acteurs pour penser le passé. La narration proposée par cette figure majeure de l'Institut nous permet d'inscrire ce récit dans le temps long de l'histoire des échanges diplomatiques et commerciaux franco-chinois.

Prolongement français du Traité de Nanjing qui clôturait la première Guerre de l'opium entre l'Empire des Qing et la Couronne britannique, le Traité de Whampoa de 1844 contraignait les Mandchous à concéder une série de droits commerciaux, militaires et juridiques aux Français dans plusieurs ports de l'Empire du milieu. ${ }^{16}$ Point de départ d'une longue période de relations asymétriques entre les deux pays, les avantages acquis à Whampoa furent les premiers d'une série de traités franco-chinois qui allaient offrir à la France le contrôle de l'Indochine en 1885 puis l'annexion de la Baie de Guangzhou (Fort Bayard) et des droits économiques et commerciaux étendus sur la Chine du Sud-est en $1898 .^{17}$

Les dernières années du XIX siècle témoignent d'un changement d'échelle dans la politique d'expansion des puissances industrielles modernes, essentiellement européennes, américaines et japonaises. Les processus d'assujettissement et de domination coloniale des territoires et des économies non occidentales gagnent en intensité et prennent un caractère systématique qui signe l'avènement de l'impérialisme. L'accumulation provoquée par le développement rapide d'un capitalisme industriel en crise de surproduction à partir de 1873 déplace les capitaux européens vers l'Afrique, le Moyen-Orient et l'Asie. Dans le contexte d'une concurrence économique et militaire acharnée entre les puissances coloniales, ces capitaux financent les constructions de ports, de voies ferrées, l'exploitation des matières premières, le développement industriel, etc.

En Chine, à la circulation des marchandises et aux comptoirs commerciaux de l'époque libre-échangiste des guerres de l'opium succède, à partir du traité de Shimonoseki en 1895 , un impérialisme intense et multiforme qui transforme le pays en un protectorat à la merci des puissances coloniales et de leurs capitaux. Ces dernières se partagent désormais 
le territoire des Qing en zones d'influences économiques et stratégiques, annexent des « territoires à bail » et établissent des bases militaires pour défendre leurs intérêts.

Les marchands lyonnais, au premier rang desquels les soyeux, joueront un rôle économique et diplomatique actif dans l'expérience coloniale française en Asie. Comme le souligne Maurice Courant, plusieurs lyonnais, dont un représentant de la Chambre de commerce, participent à la Mission Lagrené de 1844 qui prépare la signature du Traité de Whampoa. Parmi les objectifs de la mission, outre la nécessité pour la France de ne pas laisser l'Angleterre s'emparer seule du marché chinois, il y avait celui d'établir des relations commerciales avec la Chine afin de permettre aux soyeux français, et surtout lyonnais, à la fois d'écouler leurs produits finis et de s'approvisionner matière première. ${ }^{18}$ $\mathrm{Au}$ cours de la seconde moitié du XIX $\mathrm{X}^{\mathrm{e}}$ siècle, tandis que la « maladie du ver à soie contraint les industriels lyonnais à importer leur matière première de Chine et du Japon ", les soyeux lyonnais vont s'efforcer de renforcer leurs échanges commerciaux avec l'Asie. ${ }^{19}$ La Condition des soies de Guangzhou avait été créée en 1860 avec le concours de la Chambre de commerce de Lyon. A la fin du XIXe siècle, la moitié des exportations de soie depuis Shanghai sont écoulées vers la région lyonnaise. Témoins de cet intérêt des industriels lyonnais pour l'Asie du sud-est et la Chine, trois missions commerciales lyonnaises de longue durée partiront en Asie au cours des dernières décennies du XIX ${ }^{\mathrm{e}}$ siècle. En 1896, le ministre d'Etat Li Hongzhang 李鸿章 réalisera une visite diplomatique à Lyon, accompagné de quelques étudiants chinois en ingénierie et agronomie. ${ }^{20}$

A la suite des recommandations de la dernière mission d'exploration commerciale (1895-1897), sur laquelle nous allons revenir, un enseignement de langue chinoise fut établi à Lyon en 1900. Le compte-rendu de la mission soulignait l'importance de la connaissance des « indigènes » dans la perspective d'une expansion économique française en Chine et en Asie. ${ }^{21}$ Le Gouverneur général de l'Indochine française, Paul Doumer, dans une lettre au président de la Chambre de commerce de Lyon, le 10 janvier 1899, insista sur la nécessité de créer un enseignement de langue chinoise et de langue annamite à Lyon et proposa de participer au financement de ce qu'il nommait un « enseignement colonial » destiné à servir les œuvres commerciales de la colonisation et à former des sujets pour l'Indochine française et les pays soyeux d'Extrême-Orient. ${ }^{22}$ L'Ecole coloniale de Lyon, qui deviendra l'Ecole de préparation coloniale en 1924, fut fondée en 1900 sur une initiative de la Chambre de commerce de Lyon, et notamment de l'industriel lyonnais Ulysse Pila (1837-1909). ${ }^{23}$ C'est dans le cadre de cette école que Maurice Courant deviendra le premier professeur de langue chinoise de Lyon dans le cadre de l'Ecole coloniale. ${ }^{24}$ Une chaire sera créée en 1913. Ces éléments factuels soulignent la relation intime entre l'enseignement du chinois à Lyon et l'Empire colonial français.

\section{« L'aptitude au progrès du Chinois »}

19 C'est à l'initiative d'Ulysse Pila que la Chambre de commerce de Lyon monte la « Mission lyonnaise d'exploration commerciale en Chine » en 1895. Si cette mission peut constituer un jalon dans la généalogie de l'Institut, ce sont surtout les connivences idéologiques avec le projet de l'IFCL qui méritent d'être soulignées. Les lignes qui suivent sont empruntées aux conclusions du compte-rendu détaillé de la mission. Si les deux tomes de ce rapport visaient à présenter les capacités de production et de consommation des Chinois, ils constituaient aussi un récit de voyage, une description ethnographique et un tableau économique précis de la Chine du sud. ${ }^{25}$ 
L'aptitude au progrès du Chinois.

Le Chinois est un "homme économique » des plus intéressants. Il a évidemment, pour diverses causes (grande diversité de climat, d'où énergie de la race; civilisation spéciale, mais étonnamment complète et même compliquée dans son genre; - absence totale de scrupule religieux; - intelligence aiguisée et grande capacité de travail) infiniment plus de besoins que l'Arabe et le Nègre par exemple ; et il est susceptible d'en acquérir bien davantage (...)

Le Chinois ne changera probablement pas certains détails de son organisation économique, appropriés au climat; il conservera les ameublements de bois dur par exemple. Mais, au moins dans le Nord, et en hiver, je ne vois pas du tout pourquoi il n'apprendra pas à apprécier les draps de lit et les couvertures de laine, quand il pourra les acheter. Il mettra certainement, à la même échéance, des vitres à ses maisons. C'est actuellement un des premiers soins et une des fiertés du Chinois enrichi. Et ainsi de suite. On pourrait multiplier les exemples. Il suffit de parcourir la liste des vingt-deux articles divers, relevés à l'entrée de Chang-hai, et cités plus haut, depuis le champagne jusqu'aux boites à musique et des jumelles aux lanceparfums, pour être persuadé que le Chinois réserve bien des surprises au vieux monde.

C'est de ce Chinois, enrichi par une exploitation rationnelle des ressources de son sol, sous la direction inévitable et avec les capitaux indispensables des vieux pays ; et non pas du Chinois actuel qui vit avec fr. 0, 35 par jour, que l'industrie européenne a besoin. ${ }^{26}$

20 Avec le privilège de notre position historique contemporaine, cette vision du futur de la Chine, et du « Chinois », est fascinante en ceci qu'elle apparait prémonitoire. La lecture de ce texte à partir du présent laisse entrevoir l'avènement d'une société ordonnée par le capitalisme de la consommation tel qu'il se développera au Etats-Unis a partir de la fin de la première guerre mondiale pour s'étendre ensuite au reste du monde au cours du XX siècle.

21 Cet extrait est remarquablement explicite dans son dévoilement d'une géographie humaine et d'un regard historique profondément imprégnés d'une épistémologie coloniale eurocentrée. Il révèle l'articulation décisive entre deux discours massifs et récurrents qui ont fortement contribué à instituer le «sujet chinois moderne»: la racialisation de l'identité et l'historicisme.

Dans le contexte européen des années 1920, cette catégorie instituée du « Chinois » s'est trouvée réifiée par l'idéologie du racialisme scientifique, dominante en France et en Europe à partir de la seconde moitié du XIX siècle. ${ }^{27}$ Les auteurs s'inspirent d'un biologisme qui se propose non seulement de classifier les individus - au moyen de typologies physiologiques et psychologiques - mais aussi de les hiérarchiser: le «Chinois» ayant «infiniment plus de besoins que l'Arabe et le Nègre ». La substantialisation $\mathrm{du}$ «Chinois » est ici révélée par l'article défini et l'emploi du verbe être au présent : « le Chinois est...».

23 A cette lecture différentialiste du monde qui pose l'identité raciale comme un fait de nature, se superpose le métarécit historiciste, où l'idée qu'il existerait des lois, des tendances générales qui détermineraient le développement des sociétés humaines : « une succession d'étapes déterminées par des évènements censés s'être toujours d'abord produits uniquement en Europe de l'Ouest $»{ }^{28}$ Lieu de production du discours historiciste, l'Europe (l'Occident) se situait à la pointe de l'histoire et convertissait les différences culturelles avec les « Autres » en écart historique. ${ }^{29}$

En soulignant « l'aptitude au progrès du Chinois », les rapporteurs de la mission lyonnaise « confirment » que le "Chinois » appartient à un certain type d'homme, une " race », 
capable de progrès, adaptable dans le monde moderne. Le déterminisme se signe ici par l'usage du futur pour évoquer un devenir moderne inévitable du «Chinois». Il va s'approprier les nouvelles technologies («l'énergie électrique»), les infrastructures modernes («routes, ponts, travaux d'hydraulique agricole»), adopter une logique économique capitaliste ( une exploitation rationnelle des richesses de son sol»). Mais la définition de la modernité ne se limite pas ici à des conditions économiques. La métamorphose $d u$ "Chinois» sera aussi culturelle: elle vise ses habitudes, ses comportements, son mode de vie. Dans la logique libérale des auteurs du rapport, ce futur moderne verra l'avènement d'un homo-oeconomicus «Chinois » mû par ses intérêts et en quête de nouveaux besoins.

L'énumération de ces "choses modernes " $^{30}$ que sont le "Champagne, (les) boites à musiques, (les) jumelles, (le) lance-parfum » illustre la thèse de Marx qui soulignait que la grandeur du capitalisme se trouvait dans sa capacité à remplacer des «besoins naturels » par des "besoins produits historiquement ${ }^{31}$ Au-delà de la description de nouveaux objets et de nouvelles pratiques, le texte évoque une inflexion du «Chinois » en tant que sujet susceptible de faire évoluer ses désirs: «je ne vois pas du tout pourquoi il n'apprendra pas à apprécier les draps de lit et les couvertures de laine "; "C'est actuellement un des premiers soins et une des fiertés du Chinois enrichi » que de « mettre des vitres à ses fenêtres ».

Le devenir moderne du «Chinois» ne saurait évidemment être compris comme le fruit d'une évolution naturelle et universelle, sauf à reprendre à notre compte le discours historiciste. Depuis deux siècles, la Chine s'est appropriée progressivement, et dans la douleur, le langage et les pratiques d'une modernité eurocentrée : la techno-science, la maitrise prétendument «rationnelle» de la nature et des activités humaines, le capitalisme, les institutions de l'Etat-nation. Cette mutation de la Chine peut être élucidée, au moins partiellement, en considérant la domination épistémologique, technologique, économique et politique globale des sociétés euro-américaines sur la société chinoise. Elle est identifiée par les auteurs du rapport comme un projet conscient de transformation visant les conditions économiques de la Chine et, plus profondément, les comportements et les pratiques d'un « sujet chinois».

L'articulation de ce discours (sur l'Autre) avec son lieu d'énonciation est cruciale pour comprendre la dimension coloniale de la modernité. Le devenir moderne du «Chinois » constitue une nécessité pour «nous »: «l'industrie européenne », les «vieux pays ». La relation (coloniale) de pouvoir est ainsi révélée par le rôle assigné à l'Europe dans le processus de transformation du «Chinois»: "C'est de ce Chinois, enrichi par une exploitation rationnelle des ressources de son sol, sous la direction inévitable et avec les capitaux indispensables des vieux pays ; - et non pas du Chinois actuel qui vit avec fr.0, 35 par jour, que l'industrie européenne a besoin. »

Ces propos révèlent l'articulation intime entre le projet d'une transformation sociale, culturelle et intellectuelle du "Chinois» et les intérêts économiques de l'industrie européenne. Ce sont la «direction inévitable» et les «capitaux indispensables » qui permettront de faire du «Chinois actuel» un «Chinois enrichi». Si la philosophie du projet moderne est mue par une rationalité économique - les « besoins de l'industrie » -, elle implique aussi des transformations culturelles profondes.

29 Ce texte apparait représentatif de mutations décisives du discours colonial français au cours de la Troisième république. Si l'entreprise coloniale de la France en Asie du sud-est avait été initiée par le Second Empire de Napoléon, c'est la Troisième république qui 
permettra l'affirmation d'un « impérialisme colonial français » cohérent et systématique : «instrument décisif de progrès matériel et de développement moral [pour les colonisés] », le projet colonial devait aussi permettre un accroissement de la prospérité de la France. ${ }^{32}$ Le colonialisme français est passé d'une logique d'exploitation des territoires et d'assujettissement brutal des colonisés à des dispositifs d'accompagnement du développement de l'économie des sociétés sous sa domination. ${ }^{33}$

Cette nouvelle logique était certainement justifiée par l'universalisme républicain, élément moteur du projet colonial: la «mission civilisatrice» et la nécessité d'une "solidarité humaine». Cependant, l'idée que la France pourrait bénéficier du développement économique de ces contrées a constitué une évolution notable de sa politique coloniale. Les thèses du diplomate et administrateur d'empire Jules Harmand ont constitué une illustration de cette nouvelle logique. Dans Domination et colonisation (1910), il a théorisé une politique coloniale basée sur l'association, l'entraide mutuelle et le développement. ${ }^{34} \mathrm{Sa}$ "politique d'association » suggérait des rapports plus équilibrés, bien qu'asymétriques, entre le «conquérant » et le « conquis ». ${ }^{35}$ La défense des intérêts économiques et politiques du "conquérant» ne devait pas exonérer celui-ci d'une responsabilité morale sur le " conquis ». Le colonisateur devait " prendre en charge (les) âmes, (les) besoins intellectuels et (les) sentiments ataviques » du colonisé. ${ }^{36}$ Ces formes d'assujettissement qui ne réduisent pas le colonisé à un pur objet, et qui n'excluent pas une logique de transformation de ce dernier, nous éclairent non seulement sur le discours qui accompagne la mission lyonnaise d'exploration commerciale mais aussi sur le dispositif de l'Institut franco-chinois de Lyon. ${ }^{37}$

\section{Façonner des « hommes nouveaux »}

31 Cette articulation coloniale à visée humaniste entre la poursuite des intérêts économiques et politiques français et le souci paternaliste d'aider l' « Autre » se retrouve dans les discours autour de l'IFCL. ${ }^{38} \mathrm{Il}$ faut souligner que ce sont les milieux de la bourgeoisie libérale française, non catholique, radical-socialiste, héritière du rationalisme et du scientisme des lumières, portant hauts les idéaux des Droits de l'homme, qui apparaissent comme les plus actifs pour soutenir les expériences éducatives chinoises en France. Ils considèrent la question de l'instruction comme cruciale dans ce qu'ils envisagent comme un processus historique d'émancipation des individus et des peuples. ${ }^{39}$

Au retour d'une mission en Chine décisive pour la création de l'Institut, le président de la Ligue des droits de l'homme Paul Painlevé (ancien ministre de l'Instruction publique et Président du conseil) tenait un discours de collaboration et d'entraide à l'égard de la Chine et des Chinois : "Nul pays plus que la Chine n'est digne de retenir l'attention de l'Europe, mais il ne faut pas que le monde occidental considère la Chine comme un pays à exploiter, s'il en était ainsi cela finirait mal. Ce qu'il faut c'est venir en aide à la Chine et non la coloniser. » Il insistait sur la nécessité de «collaborer avec les Chinois, et non d'essayer de les dominer, car le Chinois veut, à juste titre, qu'on ait de la considération pour lui. $\star^{40}$

Cette "aide» qui allait se manifester par la formation des cerveaux chinois n'était concevable que dans la perspective d'une supériorité culturelle de l'Europe. Empruntant une rhétorique encore plus explicite, Georges Dubarbier, qui deviendra secrétaire général de l'IFCL à partir de 1935, disait « espérer que le jour n'est plus trop lointain où la Chine dégagé par elle-même de son archaïsme et en définitive éduquée par l'étranger, aura 
parmi les grandes puissances mondiales le rang qu'elle prétend légitimement tenir et auquel lui donne droit son passé historique $»^{41}$ Le caractère légitime de la domination française (occidentale) est explicite à travers cette nécessité pour la Chine d'être " éduquée par l'étranger ", et l'IFCL devient un élément de ce dispositif qui vise à orienter la société chinoise dans le sens d'une histoire imaginée par les élites politiques et intellectuelles européennes.

En rappelant les objectifs qui ont présidé à la création de l'IFCL, Maurice Courant affirmait: «L'Institut franco-chinois du Fort St Irénée est destiné à former une élite d'universitaires et de techniciens capables de diriger le développement de la Chine dans le sens qu'exigent les circonstances actuelles, aussi bien sur le terrain industriel, commercial, artistique que sur le terrain scientifique et pédagogique. $»^{42}$

Courant insistait sur la nécessité pour cette nouvelle élite de conduire la Chine « dans le sens qu'exigent les circonstances actuelles ». L'IFCL était conçu comme un dispositif qui visait à orienter le devenir de la Chine dans la direction souhaitée par les acteurs français et occidentaux. Il notait que ces « amitiés » allaient permettre « l'expansion des idées » et la « diffusion des produits français ».43 La formation de ces étudiants ne relevait pas d'un humanisme désintéressé, mais elle devait offrir à la France, à terme, un retour sur investissement : «Aujourd'hui, on nous demande la lumière : soyons sûrs que le nouveau foyer allumé sur les bords du pacifique ne tardera pas à nous renvoyer lumière et chaleur. $»^{44}$ Futurs ambassadeurs de la France en Chine, ces étudiants allaient retourner en Chine «porter la bonne nouvelle, j'entends nos idées morales et nos méthodes scientifiques $»{ }^{45}$

L'IFCL avait pour fonction d'accélérer un processus de modernisation de la société chinoise à travers la formation de sujets modernes : les étudiants venaient « compléter leur culture traditionnelle et chercher en Occident l'armature technique de la vie moderne $»{ }^{46}$ Ils rejoignaient une école de la modernité qui se confondait avec la science, les valeurs et le savoir-faire français. Il s'agissait « de donner à une élite intellectuelle chinoise une culture universitaire française ${ }^{47}$ Les promoteurs du projet insistaient sur l'inculcation d'« idées morales » et l'assimilation de "valeurs républicaines ». En 1928, le bulletin de l'Institut présentait le «Fort St Irénée » comme « la citadelle pacifique où se forment les hommes nouveaux de la Chine, le promontoire lumineux d'où ils partent en porte-flambeaux, conquérir leur vieux pays à l'humanité paisible et pensante $»{ }^{48}$

Ce souci d'œuvrer à une transformation en profondeur de la subjectivité des étudiants est explicite : "Ces jeunes gens sont à l'âge où se gravent les impressions profondes, où se nouent les amitiés durables. Les services que nous leur auront rendu, le profit tiré de leur séjour parmi nous, entreront à jamais dans leur pensées, feront partie de leur être même ». Une démarche qui fait directement écho aux idées de Jules Harmand sur la politique coloniale française lorsqu'il invoquait la responsabilité morale $\mathrm{du}$ « conquérant » prenant en charge « les âmes, les besoins intellectuels et les sentimentaux ataviques » des colonisés.

Malgré les divergences de vues et les tensions récurrentes entre partie chinoises et françaises, les initiateurs chinois de ce projet, de Cai Yuanpei 蔡元培 à Li Shizeng 李石曾 en passant par Wu Zhihui 吳稚睴ne contestent pas ce rôle assigné à l'Institut de formation d'une élite scientifique moderne. L'humanisme colonial français scientiste et universaliste s'avère parfaitement compatible avec l'imaginaire culturel et les épistémologies des intellectuels chinois à l'initiative dans ce projet. Lecteurs d'Elisée Reclus, de Kropotkine et d'Auguste Comte, Li Shizeng, Wu Zhihui ou Chu Minyi 䙋民谊 
sont, à leur arrivée en France, des anarchistes cosmopolites, épris de science et de progrès, amoureux de la France de la Révolution française et de son idéal républicain. ${ }^{49} \mathrm{La}$ formation intellectuelle et scientifique des étudiants chinois en France doit permettre à la Chine de devenir une civilisation moderne et avancée. ${ }^{50}$ C'est l'objectif commun - malgré les divergences en termes d'organisation, de recrutement des étudiants et de finalité du projet - assigné à toutes les expériences éducatives chinoises en France au cours des premières décennies du vingtième siècle: de la fameuse coopérative libertaire "Caséosojaïne $\aleph^{51}$ de Li Shizeng à l'IFCL en passant par les initiatives malheureuses de la Société franco-chinoise d'éducation qui accueillera près de 1550 étudiants chinois entre 1919 et 1921. ${ }^{52}$ Avec l'Institut franco-chinois de Lyon, les idéaux anarchistes et cosmopolites des premières initiatives éducatives tendent à s'effacer devant une instrumentalisation plus évidente de la science au service de la construction nationale.

\section{Devenir moderne, rester Chinois}

Le devenir moderne de ces étudiants ne saurait se comprendre uniquement à travers les discours d'intention des acteurs et des concepteurs du projet. Il s'incarne aussi dans les pratiques du quotidien de ces étudiants : " Les étudiants chinois vivent au fort St Irénée avec un sens du confortable directement inspiré des collèges d'Oxford ou d'Harvard. Electricité, eau courante, salles de bains, tennis, basket-ball, cuisine européenne, concourent à leur créer la somme des plaisirs nécessaires aux diversions de l'étude. $\rrbracket^{53} \mathrm{~A}$ travers la comparaison avec ces prestigieuses universités et l'énumération de ces signes d'un confort nouveau et contemporain, ce témoignage de L'Est Républicain dévoile une modernité de la vie quotidienne de ces étudiants que les archives de l'Institut, notamment photographiques, viennent effectivement confirmer : billards, cinéma, tenues vestimentaires, pratiques artistiques, sport, etc.

Bien sûr, en qualifiant cette vie, ces pratiques et ces objets de « moderne » nous utilisons une catégorie épistémologique dont nous souhaitons par ailleurs dévoiler les usages idéologiques. La critique postcoloniale a révélé que l'usage discursif de l'adjectif « moderne ", en tant que propriété exclusive de l'Europe, fut un élément constituant, et légitimant, de l'impérialisme européen. Cependant, la reconnaissance, et la réification, de différences culturelles évidentes entre des contextes sociaux-historiques distincts n'est pas une figure suffisante pour qualifier une historiographie eurocentrée. Sa signature apparait lorsque l'interprétation de ces différences s'effectue à partir d'une conception linéaire, universelle et hiérarchisée du temps et de l'histoire dans laquelle le moderne se confond systématiquement avec l'occidental.

41 Notre propos ne vise pas à nier que les pratiques, les objets et les imaginaires auxquels se confrontent les étudiants en arrivant en France peuvent être qualifiés de nouveaux, d'inédits, de différents eu égard à l'univers socioculturel dans lequel certains d'entre eux ont grandi. ${ }^{54}$ Les pratiques discursives autour de l'IFCL s'inscrivent dans cette épistémologie binaire du discours orientaliste où la France (l'Occident/l'Europe) se confond avec une modernité scientifique, intellectuelle, technique, mais aussi morale et politique tandis que la Chine est certes une ancienne civilisation, parfois envisagée comme prestigieuse, mais qui nécessite maintenant d'être transformée, éduquée et modernisée. "Chinois » n'a ici peu d'importance - se définit toujours dans un miroir inversé : local, 
traditionnel et non-moderne. Dès lors, au discours historiciste se trouve toujours associé, manifeste ou latent, le discours identitaire, en tant que réassurance de la différence culturelle. Si les étudiants se situent dans un cadre moderne/occidental qui doit participer à la transformation de leur être, leur identité primordiale ne s'évanouit pas pour autant : «Des chambres, à deux lits, proprettes et décorées avec le soin minutieux qui caractérise l'Oriental, abritent par affinités, deux étudiants de la même classe. » La mention du sujet est discrète mais signifiante : c'est un "Oriental » et, à ce titre, il est pourvu de caractéristiques spécifiques à son identité, en l'occurrence ici la minutie. Comme celle du «Chinois », la catégorie de l' « Oriental » est une signification imaginaire sociale instituée dans la France de la première moitié du vingtième siècle..$^{55}$

Dans cette fabrique des subjectivités modernes qu'est l'IFCL, les étudiants sont interpellés en tant que "Chinois ", signifiant qui renvoie à une ethnicité solidifiée par le racialisme scientifique, la notion de nationalité et un culturalisme orientalisant. La culture moderne, qui doit "pénétrer leur être même », n'annule pas le noyau essentiel de l'identité de ces étudiants. L'" armature technique de la vie moderne » offerte par l'Occident s'impose à une identité persistante. Le discours est ainsi circonscrit dans ce cadre binaire et asymétrique: devenir moderne, rester "Chinois", c'est le leitmotiv dominant du dispositif qui contribue à l'émergence de ces "sujets chinois modernes", parfaitement illustré dans ce commentaire journalistique sur l'exposition de peinture des étudiants en 1930:

Les artistes ont salué le renouveau et leurs œuvres sont modernes avec un je ne sais quoi qui sent malgré tout le respect atavique des traditions passées.

Il y a une joie à constater là, comme dans les autres domaines de l'esprit le triomphe de la culture française.

Il y a tristesse à voir s'évanouir en fumées vaporeuses les fantômes de la vieille Chine, les mandarins solennels, les idoles bouddhiques, les guerriers aux armures contournées, les pagodes aux angles relevés comme des sourires. ${ }^{56}$

C'est le récit de la métamorphose culturelle de ces étudiants. Le dispositif fonctionne. Tandis que la «culture française " "triomphe » et que la "vieille Chine" s'efface, les formes du « moderne » s'imposent inexorablement devant celles, anciennes, de la Chine. Les usages normatifs et historicistes de ce binôme "moderne/traditionnel » s'inscrivent dans le « déni de contemporanéité » auquel furent assignées les sociétés non-occidentales pendant le temps long de l'expansion coloniale européenne. ${ }^{57}$

Pourtant, un noyau identitaire, «Oriental » ou «Chinois », résiste, un « je ne sais quoi » de « respect atavique des traditions passées », un enracinement héréditaire de ces gens-là dans une identité essentialisée. Ce « je ne sais quoi » constitue ce que Rey Chow nomme le "supplément ethnique » qui colle, inévitablement, à la peau de ces individus. ${ }^{58}$ Alors même que le dispositif voudrait transformer «leur être même ", ils n'échappent pas à leur condition chinoise, prisonniers de leur sinité.

\section{La valeur ajoutée de l'ethnicité}

Cette articulation entre le grand récit de la modernité eurocentrée et une assignation à résidence identitaire se trouve confirmée par la manière dont ces étudiants s'inscrivent dans le discours scientifique et les institutions du champ académique français, autre dimension centrale du dispositif. Dans une étude précédente, nous avions traité du thème de la subjectivité et du lieu d'énonciation dans les thèses de doctorat des étudiants de 
l'institut. Il s'agissait d'éclairer le rapport que ces étudiants entretenaient avec le terrain de leur recherche, la "Chine ", qu'ils se représentaient aussi comme étant leur communauté d'appartenance. L'analyse des paratextes démontrait que ces écrits se trouvaient affectés par l'affirmation d'une subjectivité nationale, ethnique et culturelle. Nous avions lu dans ces textes un "discours chinois ", latent ou manifeste, qui interrogeait les principes d'objectivité et de neutralité assignés à la littérature scientifique. Absente, voilée ou à la marge dans certaines thèses, cette « sinité » se voyait revendiquée et valorisée dans d'autres, tantôt comme une condition culturelle, celle d'une relation privilégiée, intime, celle du " natif ", avec l'objet d'étude, tantôt comme une position politique, manifestation affirmée d'un sentiment patriotique ou nationaliste. En établissant des liens entre les productions intellectuelles et les localisations géohistoriques, les pratiques théoriques postcoloniales ont ébranlé l'épistémologie moderne occidentale, qui « embaum(ait) son propre lieu d'énonciation et projet(ait) une idée de la connaissance comme schémas universaux à partir d'une histoire locale particulière et masquée $»{ }^{59}$ Historiquement, ce privilège épistémique, ou l'exclusivité de l'objectivité scientifique rendue possible par une confiscation de la position universelle, a développé une asymétrie structurelle dans la distribution du travail scientifique entre le monde euro-américain en tant que lieu de production de connaissances et le reste du monde, réduit à la condition d'objet d'étude.

Lorsque les premiers étudiants de l'Institut franco-chinois de Lyon obtinrent leur diplôme de doctorat à la fin des années mille neuf cent vingt, la Chine se situait au sein du « reste du monde » dans cette géopolitique coloniale de la connaissance. Imaginée comme non moderne et « en retard ", la société et la culture chinoise, ainsi que son ancienne haute culture mandarinale, constituaient des éléments d'une civilisation "intéressante à comprendre et à étudier » en tant qu'objet d'étude, mais incapable d'être un espace de production de connaissance. Le lieu - cadre théorique, institutionnel, langue, géographie d'où s'énonçait une pensée académique et scientifique moderne ne pouvait être situé que dans les sociétés euro-américaines.

Dans ce contexte, le cas de l'IFCL nous confronte non seulement à un déplacement géographique et culturel mais aussi épistémologique des étudiants. Les pensionnaires thésards de l'Institut se sont retrouvés dans la position de producteurs de connaissances assujettis aux normes disciplinaires de la science moderne/occidentale dans l'espace imaginé comme rationnel et autonome de la culture académique française. Devenir moderne, c'était aussi acquérir le droit de parler à partir d'une position envisagée à l'époque comme universelle et transcendante.

50 Sur les 136 thèses recensées à ce jour, 41 d'entre elles portent sur un thème en relation avec la Chine. Les sujets traités couvrent toutes les disciplines, et nombreuses sont les thèses qui font référence à la situation contemporaine chinoise. Ce ratio significatif témoigne du choix chez une partie non négligeable des étudiants et/ou de leurs directeurs de recherche de prendre la Chine comme objet d'étude. La dichotomie récurrente entre "Chinois-sujet de la connaissance» et «Chine-objet de la connaissance » semble confirmée par l'importance du critère de l'ethnicité dans le travail de production scientifique. Pire, l'ethnicité apparait souvent en tant que valeur ajoutée à la connaissance.

51 Le biais est explicite chez Maurice Courant dans sa présentation d'une collection d'ouvrages dédiée aux meilleurs travaux des étudiants de l'Institut. Tandis qu'il loue la 
capacité des étudiants à s'approprier les «méthodes scientifiques occidentales ", Courant insiste sur leurs qualités intrinsèques de natifs susceptibles d'offrir au lecteur français une meilleure connaissance de la « civilisation chinoise » : « Faire connaitre en France la civilisation chinoise par les travaux mêmes des Chinois, n'est-ce pas une voie naturelle vers ce but? (...) Nos jeunes amis chinois nous aideront, avec leurs qualités natives; nous le souhaitons. ${ }^{60}$

Le savoir du natif est aussi souligné par Edmond Goblot dans sa préface à la thèse de philosophie de l'étudiant Hoang Tsen-yue 黄曾樾. Après avoir noté qu'il y a «peu de chances pour qu'en procédant par conjectures, un européen $d u 20^{e}$ siècle de l'ère chrétienne rejoigne la pensée d'un philosophe extrême-oriental du $5^{\mathrm{e}}$ ou $6^{\mathrm{e}}$ siècle avant l'ère chrétienne ", le philosophe français interpelle son étudiant en ces termes : "Mais vous devez être en mesure, vous Chinois, de rechercher si l'étude détaillée des textes, aidée au besoin par la philologie, confirme, condamne ou rectifie cette hypothèse ${ }^{61} \mathrm{En}$ quête d'une interprétation «chinoise » des textes classiques, Goblot établit une position de continuité entre un " philosophe extrême-oriental du $5 \mathrm{e}$ ou $6 \mathrm{e}$ siècle avant l'ère chrétienne » et un étudiant chinois du XXe siècle. Cette continuité est rendue possible par l'idée d'une ethnicité chinoise transhistorique. Ce discours met en lumière un rapport non problématisé entre une hypothétique identité chinoise essentialisée et des textes écrits dans une langue considérée de manière a-historique comme chinoise. ${ }^{62}$

L'opposition entre le savoir intime du natif et à la position d'extériorité du sinologue est partagée par les étudiants eux-mêmes. C'est en invoquant l'écart culturel, linguistique, spirituel, entre les spécialistes français du poète des Tang et le poète lui-même que Hsu Sung-nien 徐颂年, dans sa thèse de doctorat, tente d'expliquer la méconnaissance de la " vraie personnalité » de Li Bai en France. Notant que « certains écrivains auraient évité des erreurs à son sujet s'ils avaient mieux pénétré la langue chinoise littéraire et la vie chinoise intime », Hsu ajoute que la « simple compréhension ne suffit pas »:

Il faut sentir. Comment, par exemple, sentir un poème écrit dans notre langue sans avoir vécu de longues années dans l'intimité de chez nous? (sic.) Les images peintes vous sont-elles familières ? Ressentez-vous l'émotion, souvent subtile, émanant d'un poème ? Ceux qui possèdent imparfaitement le chinois littéraire, ceux qui, ayant peut-être vécu à l'écart des Chinois, défigureront, malgré qu'ils en aient, l'œuvre du poète (sic.). ${ }^{63}$

Dans la littérature universitaire, le « nous » renvoie à la communauté scientifique, à l'idée d'une coproduction de connaissances, c'est un « nous " professionnel qui masque le « je » dans un souci d'objectivité et permet à l'auteur de parler à partir d'une hypothétique position collective. Dans l'extrait ci-dessus, l'usage des pronoms " notre " et " nous " positionnent le chercheur à l'intérieur d'une communauté non plus disciplinaire et académique mais ethnique, culturelle et linguistique. Hsu Sung-nien rejette la position d'extériorité propre à la culture académique moderne pour faire corps avec son objet d'étude, le poète Li Bai. Dans une opposition classique entre sensibilité orientale et rationalité occidentale, Hsu Sung-nien revendique un savoir intime, intuitif, une perception sensible appartenant au registre de l'émotion, inaccessible aux « sinologues, essayistes, romanciers et journalistes ", bref aux érudits non chinois, " ceux ayant vécu à l'écart des Chinois ». Le savoir du " natif chinois » ne correspond pas à la connaissance rationnelle et scientifique, réservée à la " culture académique ", qu'il s'approprie en France. 

condition d'essentialiser une identité culturelle et linguistique chinoise en postulant une relation de continuité entre le monde lettré de la dynastie des Tang et l'espace linguistico-culturel contemporain de Hsu Sung-nien. Son raisonnement ne tient que dans la mesure où l'étudiant postule l'existence d'une communauté culturelle, linguistique et raciale a-historique, essentialisée à laquelle appartiennent Li Bai et Hsu Sung-nien : « la Chine ». Cette ethnicité fictive transhistorique est un élément crucial du dispositif qui contribue à la fabrication du sujet chinois moderne.

L'imaginaire essentialiste se joue des anachronismes en occultant le caractère moderne de l'identité nationale et en projetant dans les profondeurs historiques le discours contemporain de l'homogénéité culturelle et raciale de la communauté nationale. Cette sinité revendiquée par Hsu Sung-nien est le produit d'un contexte historique dépassant l'Institut Franco-chinois de Lyon - de discours, d'une normativité et d'institutions politiques et culturelles participant depuis la fin du XIX siècle à la construction de l'Etatnation chinoise moderne - qui contribue à fabriquer ces étudiants en tant que sujet national chinois.

\section{Le sujet patriote}

Lagique primordialiste de cette ethnicité fictive donne le crédit nécessaire à la diffusion de l'idéologie nationaliste moderne. Guidé par la mission éminemment politique de formation d'une élite scientifique au service de la nation chinoise, le projet de l'Institut franco-chinois de Lyon était, par essence, patriotique. Et si la conscience politique et la ferveur patriotique des étudiants de l'Institut s'avèrent plus discrètes que celles de leurs compatriotes parisiens très militants du Mouvement Etudes-Travail, le patriotisme n'en reste pas moins une réalité attestée par plusieurs travaux historiques. ${ }^{64}$ En symétrie avec le discours de l'institution qui annonce que sa vocation est de fabriquer des cadres scientifiques modernes au service de la Chine de demain, l'ambition intellectuelle qui guide les étudiants s'avère souvent intimement liée au destin national.

Dans leurs travaux de recherche, ils n'expriment pas seulement une différence culturelle, ils affirment aussi une conscience nationale centrée sur les enjeux géopolitiques contemporains : de la communauté ethnique à la communauté politique, l'objet d'étude se confond toujours avec l'étudiant sujet national. Le souci de la patrie et de son salut s'avère crucial dans le choix et le discours de justification des sujets de thèses. Un nombre non négligeable de leurs travaux portent sur des problématiques sociales, juridiques, économiques et politiques de la Chine de l'époque : la réforme du système éducatif, le fonctionnement de l'administration locale, la constitution chinoise, les relations internationales de la Chine, le conflit sino-japonais, etc.

L'engagement patriotique se trouve explicitement mentionné dans de nombreuses thèses, dévoilant une tension entre le discours de l'objectivité scientifique et le souci d'affirmer une position, une appartenance au corps national, à son destin et à sa cause. Dans une thèse sur les conséquences des traités inégaux, thème politiquement sensible à l'époque, Henri T.T Tchai (Zhai Junqian 錐俊千) souligne la nécessité, mais aussi la difficulté, d'une approche objective de son sujet :

Qu'on nous permette d'ajouter que, dès le début de notre travail, tous nos efforts ont tendu à un but exclusivement objectif, d'après une documentation aussi impartiale que possible.

Transtext(e)s Transcultures 跨文本跨文化, 9 | 2014 
Mais nous tenons à ce que l'on sache aussi que, dans l'accomplissement de cette tâche, il a fallu, à maintes reprises, faire taire en nous la voix du patriotisme qui bien souvent protestait avec indignation contre la tutelle imposée à notre malheureux pays par des traités où la justice n'avait aucune part. ${ }^{65}$

La position de Tchai confine à la schizophrénie et la voix de son double, patriote convaincu, se trouve étouffée par les contraintes de l'écriture académique : «faire taire en nous la voix du patriotisme ». D'autres étudiants affirment au contraire sans complexe leur engagement, tel Li Tsi Gziou (Li Ji'ou 李濟歐), dont la thèse de médecine militaire sur "la défense passive des populations civiles contre la guerre aéro-chimique en Chine » porte sur un sujet éminemment d'actualité dans la Chine de 1933, après les bombardements japonais de Shanghai :

Il importe donc de prévoir une grande guerre entre les deux grands peuples jaunes d'ici quelques années, guerre à prédominance aérochimique ainsi que nous essaieront de le démontrer par ailleurs. Le vieil Occident étant le berceau de la culture moderne qui a si bien réussi à nos voisins sans scrupules, à l'aube du réveil de la Chine ainsi que de sa reconstruction, nous avons élaboré ce modeste travail concernant les mesures de défense à prendre contre ce péril aérochimique en nous inspirant des grands maitres européens. ${ }^{66}$

61 C'est une thèse appliquée que rédige ici Li Tsi Gziou, insistant sur la nécessité de se servir de la culture moderne du «vieil Occident» pour vaincre l'ennemi japonais. Aucun détachement, aucune mise à distance de l'auteur, mais, au contraire, une volonté assumée et affirmée de mettre la science et la recherche au service de la situation politique et militaire de la Chine. Dans son étude sur la Constitution de 1923, James Woo (Wu Kaisheng 伍凱聲) précise que sa dissertation ne peut pas être purement spéculative et doctrinale "pour un Chinois », et que sa "portée pratique » est évidente. ${ }^{67} \mathrm{Il}$ se définit comme un " étudiant chinois des Universités d'Europe » et, par ce geste, pointe lui aussi le caractère particulier de sa situation, insistant sur sa qualité de sujet national chinois au sein de la culture académique européenne. Cette position ambivalente d'« étudiants chinois des Universités d'Europe " vient déstabiliser la fausse neutralité de la culture académique, son illusoire universalité et son artificielle indépendance par rapport au lieu de la production disciplinaire, donnant à voir ce que cache le discours dominant de désintéressement et de pur savoir. ${ }^{68}$

62 Nous voyons ici comment la situation géoculturelle et historique de ces étudiants, comment leur conscience nationale, dont la construction est assurément récente à l'époque, créent une situation d'énonciation ambivalente lorsqu'ils prennent pour sujet d'étude la Chine : ils sont à la fois dedans et dehors, observant et observés, sujets et objets de la connaissance, dégagés et engagés. ${ }^{69}$

63 Là où les chercheurs américains ou européens travaillant sur la Chine ou «l'Orient » n'envisageaient pas la situation historique particulière de leur lieu d'énonciation, persuadés d'être dans l'espace neutre et universel du savoir, en situation d'extériorité avec leur objet d'étude, l'étudiant chinois de l'Institut, malgré son souci de neutralité scientifique, se trouve directement confronté à cette ambivalence.

Dans les paratextes de leurs thèses, les étudiants de l'IFCL établissent directement une relation épistémique entre leur localisation géo-historique et leur objet d'étude. Ils révèlent ainsi l'impossibilité d'une énonciation hors-sol, qui ferait abstraction de leur condition historique. Au «nous» conventionnel et professionnel qui voudrait signifier une neutralité de la culture académique et une étanchéité entre le sujet et l'objet de la connaissance, les étudiants substituent un sujet d'énonciation collectif extérieur à la 
communauté scientifique inscrit dans un contexte social, historique, culturelle et géopolitique.

\section{Hétérotopie : discours, pratiques et imaginaire national}

Dans son célèbre opus sur le nationalisme, Benedict Anderson insiste sur la représentation moderne d'un «temps vide et homogène» pour comprendre les communautés imaginées que sont les nations. ${ }^{70}$ Le développement d'un capitalisme de l'imprimerie en langues vernaculaires a rendu possible l'idée d'une temporalité synchrone entre des êtres dispersés sur un territoire étendu. Les archives de l'Institut dévoilent cette temporalité simultanée qui relie cette diaspora étudiante avec le territoire chinois. A travers le suivi de l'actualité chinoise ou les rituels civiques (fête nationale), l'Institut vit au rythme de la communauté nationale : nous sommes en France et, pendant ce temps-là, en Chine, il se passe des choses qui nous concernent et qui nous touchent. ${ }^{11}$

Pour Anderson, cette relation qui s'établit entre les membres de la communauté nationale est « imaginée » car «même les membres de la plus petite des nations ne connaitront jamais la plupart de leurs concitoyens ", à la différence d'une communauté familiale ou villageoise. Au contraire, la petite communauté étudiante de l'IFCL n'est pas imaginée, ou imaginaire, elle est concrète, réelle, à taille humaine, limitée aux murs du Fort St Irénée. Dans cet espace-temps compressé, il se reproduit à l'échelle moléculaire des pratiques, des rituels institutionnels et des discours qui viennent renforcer la conscience d'appartenance à une même communauté, et accompagnent des processus de subjectivation. Certains témoignages d'étudiants sur leur vie quotidienne à l'Institut illustrent l'efficacité d'un dispositif de nationalisation des pratiques et des discours.

Si une conscience nationale se manifeste bruyamment dans les grandes métropoles de la côte est à l'occasion des manifestations du 4 mai 1919, au moment de la création de l'IFCL, la Chine n'a pas encore inventé sa culture nationale, c'est-à-dire une culture moderne applicable de façon homogène à l'espace et à la population d'un Etat-nation. ${ }^{72}$ Fragiles réalités institutionnelles, la "république » et la «nation" sont, à l'époque, des néologismes récents désormais bien introduits dans le langage, mais la Chine n'existe pas encore en tant qu'Etat-nation moderne constitué, il lui manque notamment l'attribut, toujours très relatif et politique mais néanmoins essentiel à l'imaginaire de la nation, qu'est l'homogénéité. Politiciens et intellectuels peuvent soutenir que la nation chinoise est une substance naturelle grâce à la catégorie abstraite et fallacieuse de la "race ", mais, sur le plan des pratiques culturelles et linguistiques, la diversité est encore manifeste.

Avant de rejoindre le Fort St Irénée, si les étudiants ont en commun leur participation au processus de sélection et de formation dans les écoles préparatoires en Chine, les origines géographiques, les langues et les pratiques culturelles des étudiants nous révèlent la forte hétérogénéité initiale de cette minorité d'élus. L'étudiant Cui Zaiyang 催載陽confirme cette réalité en livrant ses souvenirs : « nous venions de régions différentes, parlions des langues différentes, avions des habitudes quotidiennes différentes $» .{ }^{73}$ Ceux de Zheng Yanfen, étudiant à l'Institut entre 1926 et 1932, permettent de mieux comprendre les effets de ce dispositif sur les pratiques des étudiants. Dans un texte écrit à l'occasion du $110^{\mathrm{e}}$ anniversaire de la naissance de Wu Zhihui (co-fondateur de l'IFCL), ce 
mathématicien, qui deviendra ministre de la Justice à Taiwan, raconte son voyage vers la France et ses premières heures au Fort St Irénée. Originaire de la région de Canton, les lignes suivantes évoquent ses difficultés de communications en Chine et à l'Institut :

Cela peut paraitre surprenant, mais la langue nationale, je l'ai apprise en France auprès d'autres étudiants de l'Institut. Je me souviens qu'avant de partir en France, alors que je me trouvais à Shanghai pour faire mes papiers, lorsque je souhaitais faire des achats, je prenais le bus pour la Rue du Sichuan où se trouvait une boutique dont le vendeur comprenait le cantonais, c'était le seul moyen pour moi de communiquer sans contraintes [...] Après notre arrivée à Lyon, les étudiants de l'Institut, notamment ceux de l'antenne internationale de l'Université Sun-yat sen, ont organisé une cérémonie d'accueil pour notre arrivée. Je devais prononcer quelques mots de remerciements. Très intimidé, j'ai pris mon courage à deux mains pour dire quelques mots en langue nationale, mais, après quelques phrases, je n'ai pas eu d'autres solutions que de m'exprimer en cantonais et de laisser un camarade effectuer la traduction. Cet événement m'avait fait comprendre quelque chose d'important : il me serait impossible de remplir mes missions sans savoir parler la langue nationale; Dès lors, je fus déterminé à l'apprendre. ${ }^{74}$

Ce texte nous rappelle que la différence entre langue maternelle et langue nationale était (est ?) une réalité dans la Chine de la première moitié du vingtième siècle. La «langue nationale », ou guoyu 國語, est un idiome officiel institué par l'Etat qui, à l'époque, vient à peine d'être imposé dans les manuels scolaires d'un système éducatif universel encore balbutiant. ${ }^{75}$ En soulignant la nécessité d'une traduction entre les étudiants, Zheng Yanfen fait l'expérience d'une discontinuité linguistique interne à la communauté nationale, reflet d'une hétérogénéité culturelle réelle. Cet épisode difficile face à ses camarades aurait été le révélateur d'une prise de conscience. Il ne serait pas en mesure de jouer son rôle de sujet chinois moderne sans la maîtrise de l'idiome commun : «il me serait impossible de remplir mes missions sans savoir parler la langue nationale ». C'est aussi la diversité linguistique et le processus d'apprentissage de la langue nationale qui sont soulignés dans les souvenirs de Danielle Li, fille de Li Shuhua 李書華, étudiant de l'Institut entre 1923 et 1926 :

Nombre de pensionnaires venaient du Hunan. Mon père, qui ne parlait que le dialecte de sa région, le hakka, a appris à leur contact le chinois officiel, le mandarin. A son époque, les écoles ne l'enseignaient pas encore systématiquement. Toute sa vie, il a gardé l'accent hunanais, alors qu'il n'a jamais vécu dans cette province. ${ }^{76}$

70 Laboratoire où les pratiques de la vie quotidienne tendent à s'uniformiser, l'Institut est aussi un espace où les étudiants identifient ces pratiques communes en tant que relevant de la culture nationale. Li Shuhua et Zheng Yanfen deviennent des «sujets chinois » à Lyon non seulement en tant que leurs manières d'être se conforment objectivement à celles des autres (ils parlent la même langue), mais aussi, et surtout, parce qu'ils s'approprient un "discours de la culture nationale » qui leur permet de nommer la " réalité» de ce qu'ils vivent au sein de l'Institut. ${ }^{77}$ Leur discours subsume des phénomènes, des pratiques, des individus ou des objets à l'intérieur d'une même catégorie : «la culture nationale ». L'enjeu n'étant pas ici l'origine réelle ou supposée d'une pratique culturelle mais la représentation que l'on s'en fait, et le discours dans laquelle celle-ci se trouve enchâssée.

71 L'isolement de cet espace " chinois » au cœur d'une société radicalement autre contribue sans doute à renforcer une vision organique et particulariste de leurs pratiques, provoquant un effacement réel et discursif des différences internes à cette microsociété. 
Précédent l'évocation de son désarroi face à ses difficultés de communications avec les autres étudiants, les toutes premières lignes du témoignage de Zheng Yanfen recouvrent les différences et les singularités entre les étudiants : « A l'IFCL, il n'y a que des étudiants chinois, on est tous ensemble, tout le monde parle la langue chinoise, tout le monde mange chinois, tout le monde a une vie quotidienne à la chinoise ${ }^{78}{ }^{78}$ Le discours qui nationalise les pratiques est confirmé par les impressions de Su Xuelin:

A notre arrivée en France, ce pays civilisé et progressiste, nous nous sommes retrouvés toute la journée noyés dans un bain chinois. Ce que nous entendions, ce que nous parlions c'était le chinois ; ce que nous mangions, c'étaient des plats chinois ; nos chambres semblaient des chambres chinoises ; du lever au coucher, le moindre de nos gestes était chinois, comme si nous étions encore en Chine... ${ }^{79}$

Que peut signifier ici le substantif « chinois ", ou zhongguo 中國, lorsqu'il s'applique à des pratiques linguistiques ou culinaires, à un agencement de l'espace ou à des habitudes du quotidien? Une enquête sur la nature profonde de ces réalités afin d'identifier l'authenticité de leur sinité n'aurait aucun sens, sauf à accréditer l'existence d'une essence culturelle chinoise hors-sol, indépendante de tout discours et de tout contexte, dont seraient habités ces étudiants. Il faut plutôt faire l'hypothèse d'une homogénéisation de certaines pratiques dans la vie quotidienne d'une part, et, simultanément, de la présence d'un imaginaire de la culture nationale qui permet aux étudiants de se représenter ces pratiques comme «chinoises». L'expression zhongshihua 中式化 (style chinois-changer) de Su Xuelin nous révèle la représentation d'un processus dynamique de transformation des habitudes, comme si les étudiants devenaient Chinois à Lyon.

Dans une conférence de 1967, Michel Foucault avait proposé le terme d'hétérotopie, ou littéralement un " espace autre ", pour évoquer des lieux dans la société qui obéissent à des règles et à un fonctionnement unique..$^{80}$ 'hôpital psychiatrique, la maison de retraite, l'école, les églises, les parcs de loisirs, une foire ou un salon sont des hétérotopies. Il ajoutait que ces lieux autres « fonctionne(nt) à plein lorsque les hommes se trouvent dans une sorte de rupture absolue avec leur temps traditionnel ». A l'origine, une notion d'anatomie, l'hétérotopie définit une pathologie caractérisée par "la présence d'un organe ou de tissus à un endroit où ils ne devraient pas normalement se trouver ». Cette analogie médicale nous semble pertinente pour penser la position géographique, sociale, culturelle très singulière de cette institution. L'IFCL serait une hétérotopie où se reproduisait, à une échelle moléculaire, le dispositif de fabrication d'un sujet chinois moderne.

\section{NOTES}

1. Chen Sanjing Chen San-jing « Une tentative réussie de collaboration franco-chinoise en matière d'éducation : l'Institut franco-chinois de Lyon » in Jean-Louis Boully, Ouvrages en langue chinoise de l'Institut franco-chinois de Lyon 1921-1946, Lyon, Bibliothèque municipale de Lyon, 1995, p. XXV. 
2. Cité dans Philippe Yann, «L'Institut franco-chinois de Lyon : un exemple réussi de collaboration en éducation? » (Mémoire de maîtrise sous la direction de Christine Cornet), Lyon, Université Lumière Lyon 2- Faculté GHHAT, 1998, p. 23.

3. Dans son histoire diplomatique de l'IFCL, Philippe Yann avait relevé un hiatus entre les ambitions françaises et chinoises sur la finalité de l'IFCL : « Alors que les premiers imaginent déjà les Chinois se mettant à l'école de la civilisation française, ces derniers [les Chinois] rêvent plutôt d'une transformation réciproque des civilisations occidentales et orientales », op. cit., p. 26.

4. Walter Mignolo, Local Histories/Global Designs: Coloniality, Subaltern Knowledges, and Border Thinking, Princeton University Press, Princeton, 2000, pp. 283-285.

5. Florent Villard, «Lieu d'énonciation, différence culturelle et conscience nationale : La Chine comme objet d'étude dans les theses des étudiants de l'Institut franco-chinois de Lyon (1921-1946) ", in Langues, littératures et cultures franco-chinoises du XXIe siècle, Taipei, Symposium International 2010, Presses de l'Université Tamkang, 2011.

6. Nous remercions les conservateurs du fonds chinois de la Bibliothèque municipale de Lyon, Valentina De Monte et Marc Gilbert, sans qui une exploitation scientifique de ces archives serait impossible.

7. Michel Foucault définit un dispositif comme un " ensemble résolument hétérogène, comportant des discours, des institutions, des aménagements architecturaux, des décisions réglementaires, des lois, des mesures administratives, des énoncés scientifiques, des propositions philanthropiques, bref : du dit aussi bien que du non-dit ", dans " Le jeu de Michel Foucault » (1977), Dits et écrits, III, Paris, Editions Gallimard, 1994, p. 299.

8. Paul Veyne, Foucault, sa pensée, sa personne, Paris, Albin Michel, 2008, p. 172.

9. Benedict Anderson, (trad. P.E. Dauzat), L'Imaginaire national : réflexions sur l'origine et l'essor du nationalisme, Paris, la Découverte, 2002, pp. 18-21.

10. L'expression est empruntée à Yves Chevrier dans «Antitradition et démocratie dans la Chine du premier XX $X^{\mathrm{e}}$ siècle : La culture moderne et la crise de l'Etat-nation» in Mireille Delmas-Marty, Pierre-Etienne Will, La Chine et la démocratie, Paris, Fayard, 2007, p. 375.

11. Rebecca Karl, Staging the World: Chinese Nationalism at the Turn of the Ttwentieth Century, Durham, Duke University Press, 2000, pp. 3-26.

12. Nous empruntons cette hypothèse aux travaux de Naoki Sakai sur le nationalisme Japon. Il propose une définition de l'«Occident » qui permet de dépasser une interprétation essentialiste et de prendre en compte les conséquences culturelles de l'expansion de la modernité. Au-delà d'une lecture purement économique, culturelle ou géopolitique, cette catégorie désignerait ainsi une position épistémologique dominante. Lieu du pouvoir et partie intégrante d'un discours de légitimation de ce pouvoir, l' "Occident» se situe toujours en surplomb, dans la position de l'universel, où du moderne, susceptible de transcender tous les particularismes. Sakai note que l' " Occident » agit en tant que référent des peuples « non-occidentaux » pour construire leur identité culturelle et nationale. Cette approche souligne l'interdépendance profonde, ce que Sakai nomme le "schéma de co-figuration», entre les affirmations particularistes des périphéries et la figure normative centrale de la modernité appelée "Occident». Voir Naoki Sakai, Translation and Subjectivity: On «Japan » and Cultural Nationalism, Minneapolis, University of Minnesota Press, 1997, p. 61 et «Nationality and the Politics of the 'Mother Tongue'», Naoki Sakai, Brett de Bary, Iyotani Toshio (eds.), Deconstructing Nationality, Cornell East Asia Series, 2005, pp. 1-38.

13. Sur cette bibliothèque, voir Valentina De Monte, «Les collections de l'ancien IFCL, Quels services pour quels usagers? Un état des lieux pour de nouvelles perspectives ", Transtext(e)sTranscultures : Journal of Global Cultural Studies, Volume 9, 2014.

14. Annales franco-chinoises, $n^{\circ} 6,1928$, p. 46. 
15. Maurice Courant, «L'Association Universitaire franco-chinoise : origine, buts et résultats ", Annales franco-chinoises, $\mathrm{n}^{\circ} 1,1927, \mathrm{p} .1$.

16. Muriel Détrie, France-Chine : quand deux mondes se rencontrent, Paris, Gallimard/ Découvertes, 2004, p. 35.

17. Immanuel C. Y. Hsu, «Late Ch'ing foreign relations, 1866-1905 " in John K. Fairbank and Kwang-Ching Liu, The Cambridge History of China, Volume 11, Late Ch'ing, 1800-1911, Part 2, pp. 70-142.

18. Marie-Anne Privat-Savigny, Pascale Le Cacheux, Hélène Chivaley, Les prémices de la mondialisation : Lyon rencontre la Chine au 19e siècle, Lyon, EMCC, 2009, p. 6 ; Claudine Salmon, « La mission de Théodose de Lagrené et les enquêtes sur les textiles d'insulinde », Archipel, 2008, volume 75, numéro 1, pp. 167-197.

19. Daniel Bouchez, « Un défricheur méconnu des études extrêmes-orientales : Maurice Courant (1865-1935) », Journal Asiatique, Tome CCLXXI, numéro 1-2, 1983, p. 79.

20. Nora Wang, Emigration et Politique : Les Etudiants-ouvriers chinois en France (1919-1925), Paris, Les Indes Savantes, 2002, p. 77.

21. Chambre de commerce de Lyon, La mission lyonnaise d'exploration commerciale en Chine 1895-1897, deux tomes, Lyon, A. Rey \& Cie, 1898.

22. Daniel Bouchez, op. cit., p. 79. Jean-François Klein note que le soutien de Paul Doumer à la création d'un enseignement de langue chinoise s'explique par les « visées expansionnistes » de ce dernier sur le Yunnan, « La création de l'école coloniale de Lyon : au cœur des polémiques du Parti colonial », Outre-mers, tome 93, n³52-353, p. 167. 23. Ibid, pp. 147-170. Lyonnais d'adoption, homme d'affaire et diplomate, farouche défenseur de la cause coloniale en Indochine, Ulysse Pila possède plusieurs qualités capables de nous faire comprendre l'esprit qui présida à la politique d'expansion coloniale française en Asie à la fin du XIX ${ }^{\mathrm{e}}$ siècle, et notamment l'articulation entre des intérêts économiques et le projet idéologique de la colonisation. Voir la biographie de Jean-François Klein, Un lyonnais en Extrême-Orient : Ulysse Pila, Vice-roi de l'Indochine, Lyon, LUGD, 1994.

24. Ibid, p. 167.

25. Voir Muriel Détrie, France-Chine : quand deux mondes se rencontrent, Paris, Gallimard/ Découvertes, 2004, p. 54.

26. Chambre de commerce de Lyon, op. cit., pp. 450-451.

27. Voir le chapitre « Races » dans Tzvetan Todorov, Nous et les Autres : La réflexion française sur la diversité humaine, Paris, Seuil, 1989, pp. 131-235.

28. Jack Goody, Le vol de l'histoire : comment l'Europe a imposé le récit de son passé au reste du monde, Paris, Editions Gallimard, 2010, p. 168.

29. "Historicism thus posited historical time as a measure of the cultural distance (at least in institutional development) that was assumed to exist between the West and the non-West", Dipesh Chakrabarty, Provincializing Europe: Postcolonial Thought and Historical Difference, Princeton, Princeton University Press, 2000, p. 8.

30. Frank Dikötter, Things Modern: Material Culture and Everyday Life in China, Hurst \& Company, London, 2006.

31. Kostas Papaioannou, Marx et les marxistes, Paris, Flammarion, 1972, p. 118. L'Opium, à partir du début du XIXe siècle, fut un bel exemple en Chine de ces « besoins produits historiquement », en l'occurrence à l'époque par la British East India Company, voir Gregory Lee, «L'opium et le Chinois dans le discours colonialistes », Nouvelles du Sud, n³3, 2003, 35-47. 
32. Raoul Girardet, Le nationalisme français : Anthologie 1871-1914, Paris, Éditions du Seuil, 1983, p. 85.

33. Gary Wilder, The French Imperial Nation-State: Negritude and Colonial Humanism between the Two World Wars, Chicago, University of Chicago Press, 2005.

34. Jules Harmand, Domination et colonisation, Paris, Flammarion, 1910.

35. Ibid, p. 159.

36. Ibid, p. 158. Harmand notait que la politique d'association n'est efficace que lorsqu'elle s'adresse à « une population homogène et cohérente, une civilisation originale assez avancée, ayant une certaine conscience de sa personnalité et possédant un héritage historique ", p. 159. C'est une définition qui correspond assez précisément aux représentations orientalistes de la Chine à l'époque, en tant que civilisation anciennement prestigieuse mais désormais déclinante.

37. L'approche particulière des acteurs lyonnais dans le projet colonial français permet aussi de mieux comprendre l'esprit de ces phrases conclusives de la mission. Très impliqués dans les projets coloniaux en Asie en raison de l'économie de la soie, les milieux d'affaires et à la bourgeoisie lyonnaise se distinguent de Paris par une conception plus libérale de la colonisation. Ils insistent sur la dimension économique en considérant que la colonisation doit aller de pair avec un développement des régions annexées, dans l'intérêt bien compris des indigènes et des français : matières premières, main-d'œuvre mais aussi débouchés futurs pour les produits lyonnais. Voir à ce sujet Klein, op. cit., p. 150.

38. Ce que confirme Philippe Yann dans la seule étude complète à ce jour sur l'histoire de l'IFCL : « Le maintien de relations relativement régulières avec la Chine depuis le milieu du XIXe siècle a amené le développement à Lyon d'un intérêt développé pour les affaires chinoises. Toutefois, du fait de la politique d'expansion outre-mer menée par le gouvernement français durant cette même période et du caractère essentiellement commercial de ces relations, cette 'tradition chinoise' [lyonnaise] s'inscrit elle aussi nettement dans une logique coloniale », op. cit., p. 20.

39. Wang, op. cit., p. 104.

40. «Les impressions de M. Paul Painlevé, retour de Chine », Le Petit Parisien, 29/10/1920, cité dans Yann, p. 13.

41. Georges Dubarbier, «La diplomatie de la Chine nouvelle », Annales franco-chinoises, $\mathrm{n}^{\circ}$ 10, 1929, p. 18.

42. Courant, op. cit., p. 2.

43. Maurice Courant, "L'institut franco-chinois : suite et fin », Bulletin des soies et des soieries, 12/03/1921, cité dans Yann, p. 24.

44. Ibid, p. 23.

45. Ibid, p. 23.

46. Ibid, p. 23.

47. Maurice Courant, Compte-rendu du Conseil d'administration du 21/11/1921 (Tome 1, p. 15, fonds chinois, Bibliothèque municipale de Lyon).

48. Annales franco-chinoises, $\mathrm{n}^{\circ} 7,1928$, p. 25.

49. Sur Li Shizeng, voir Marianne Bastid, «Li Shizeng yu zhongfa wenhua guanxi » 李石曾與中法文 化關係 [Li Shizeng et les relations culturelles sino-françaises], Jindai Zhongguo, 126, pp. 169-176.

50. Ces objectifs sont notamment précisés dans les Statuts de la société pour les études frugales en France (1912), ou Liufa jianxue hui jianzhang 留法儉學會簡章, voir dans Shijie she 世界社, Lü'ou jiaoyu yundong 旅歐教育運動 [Le Mouvement pour l'éducation en Europe], Tours, Lü'ou zazhishe, 1916, pp. 50-51. 
51. Usine de produits alimentaires à base de soja qui offrait un travail et des cours du soir à de jeunes étudiants et ouvriers chinois.

52. Sur l'histoire de l'émigration étudiante en France au cours des années 1910s, voir Nora Wang, op. cit., passim.

53. « Lyon, carrefour des races, les Chinois de St Irénée », L'Est Républicain, 1er octobre 1930.

54. Une remarque qui n'est que partiellement valide pour les étudiants originaires des métropoles de la côte est chinoise, en particulier Shanghai. Le cinéma, la presse populaire, la mode, l'électricité, le macadam ou le confort intérieur moderne, sont autant de phénomènes communs à la société shanghaienne des années 1920.

55. Voir sur ce sujet Florent Villard, «L'Orientalisme, la Chine et les Etudes chinoises : Usages critiques et dévoiements nationalistes de la pensée d'Edward Said » in Laurent Dartigues, Makram Abbès (eds.), Orientalismes /Occidentalismes : A propos de l'œuvre d'Edward Said, Paris, Hermann, 2016.

56. «Lyon, carrefour des races, les Chinois de St Irénée », L'Est Républicain, 1er octobre 1930.

57. Johannes Fabian, Time and the Other: How Anthropology Makes Its Object, New York, Columbia University Press, 1983.

58. Rey Chow, «Introduction : On Chineseness as a Theoretical Problem » in Rey Chow (ed.), Modern Chinese Literary and Cultural Studies in the Age of Theory, Duke University Press, 2000, pp. $1-26$.

59. Nous renvoyons ici aux écrits théoriques de Walter Mignolo sur le sujet : Local Histories/Global Designs: Coloniality, Subaltern Knowledges, and Border Thinking, Princeton University Press, Princeton, 2000, p. 123. L'essai de Marie-Julie Maître dans le présent numéro étudie en profondeur cette problématique de la géopolitique de la connaissance dans le contexte de l'IFCL : "Géopolitique du savoir et philosophie dans le contexte de l'Institut franco-chinois de Lyon ", Transtext(e)s-Transcultures : Journal of Global Cultural Studies, Volume 9, 2014.

60. Maurice Courant dans les Annales franco-chinoises, $n^{\circ} 3$, trimestre 3, 1927. L'article présente la nouvelle collection Bibliotheca franco-sinica lugdunensis éditée par la Librairie Orientaliste Paul Geuthner à Paris.

61. Hoang Tsen-Yue, Étude comparative sur les philosophies de Lao Tseu, Khong Tseu, Mo Tseu, Lyon/ Paris, Rey/Leroux, 1925.

62. Dans ses travaux sur la sinité, Rey Chow décrit parfaitement ce problème d'une ethnicité fictive transhistorique construite par la sinologie et un certain regard occidental sur la Chine, voir Modern Chinese Literary and Cultural Studies in the Age of Theory, Duke University Press, 2000, pp.10-13.

63. Hsu Sung-nien, Li Thai-po : Son temps, sa vie et son œuvre, Bosc frères et M. \& L. Riou, Lyon, 1935, p. 3,4 .

64. Nora Wang, Emigration et politique : Les étudiants-ouvriers chinois en France 1919-1925, Paris, Les Indes Savantes, 2002 ; Li Chang Li, « Minguo shiqi liuxuesheng aiguo ganqing de shenghuo jichu»民國時期留學生愛國感情的生活基礎, Xuzhou Shifan Daxue Xuebao, volume 31, n³, 2005 ; Marylin A. Levine, The Found Generation: Chinese Communists in Europe During the Twenties, University of Wasington Press, 1993.

65. Henri T.T. Tchai, Essai historique et analytique de la situation internationale de la Chine, Bosc frères et M. \& L. Riou, 1929, p. 12.

66. Li Tsi Gziou, La défense passive des populations contre la guerre Aéro-chimique en Chine, Bosc frères et M. \& L. Riou, Lyon,1933, p. 12.

67. James Woo, Le problème constitutionnel chinois, Bosc frères et M. \& L. Riou, Lyon, 1925, p. 1. 
68. «Ce qui souvent en effet se fait passer comme de la science (ou est crédité de scientifique) doit son immense renommée à sa fixité et à son extraterritorialité culturelle (la neutralité). », François Laplantine, Je, nous et les autres : Etre humain au-delà des appartenances, Le Pommier-Fayard, Paris, 1999, p. 68.

69. Rey Chow avait saisit cette ambivalence dans ses travaux théoriques sur le «spectateur ethnicisé », voir Paul Bowman, The Rey Chow Reader, New-York, Columbia University Press, 2010, p. 121.

70. Il emprunte cette formule à Walter Benjamin, voir Anderson, op. cit., p. 35

71. La correspondance des étudiants, les $C R$ du Conseil d'administration ainsi que les Annales franco-chinoises témoignent de ce souci de l'actualité sociale et politique chinoise : Il y est fait mention des manifestations anti-impérialistes dans les usines de Shanghai en 1925, de l'expédition vers le nord en 1926-1927, des terribles inondations de 1931, de l'invasion japonaise en 1937, etc.

72. La haute culture lettrée, sophistiquée et homogène, sur laquelle reposait la légitimité des institutions politiques de la Chine dynastique, masquait l'hétérogénéité des populations assujettis à l'ordre impérial. Jean François Billeter évoque ces deux sphères qui structurent la société chinoise pré-moderne dans Chine trois fois muette, Paris, Allia, 2000, p. 114.

73. 《各同學來自國内各省,大家語言不同,習慣不同。。”», Cui Zaiyang, «Li ang lingyi » 里昂零 憶 [Souvenirs éparses de Lyon] in Chen Sanjing 陳三井, Qingong jianxue yundong: Zhongguo xiandai shi shiliao xuanji : 勤工儉學運動：中國現代史史料選輯 [Le Mouvement travail diligent-études frugales : Choix de documents historiques sur l'histoire moderne chinoise], Taibei, Zhengzhong Shuju Yinxing, 1981, p. 448.

74. 《說來可笑, 我的國語, 是到了法國以後跟里昂中法大學的同學學習。記得我出國 以前, 到上海去辦護照, 因為不懂講國語, 想買點東西, 坐車說到四川路懂廣東話的

店鋪去, 才能交談無阻[。。當我們到了法國里昂, 原在里昂中法大學和中山大學 海外部的同學開會歡迎我們, 我卻被推舉出來致謝詞。我不好意思推擋, 初時只好鼓 起勇氣，想勉強用國語代表新到的同學來講話，但是講了紋句，就講不同了，只好仍 舊講廣東話, 只請一位同學翻譯為國語。這一一件事給我很大的啟示 : 它說明沒有講 國語的能力便不能達成任務, 因此卻激發了我學習國語的決心。", Zheng Yanfen, 《 Huiyi jiudu zhongfa daxue, yonghuai Wu xiaozhang Zhi lao » 回憶就讀中法:大學, 永懷吳校長 稚老 [Souvenirs de mes études à l'Institut franco-chinois, en l'honneur du directeur Wu/ professeur Zhi] in Chen, op. cit., pp. 417-418.

75. Tang Tao, History of Modern Chinese Literature, Beijing, Foreign Languages Press, 1993, pp. 8-9.

76. Danielle Li, L'Eurasienne : Une femme entre Chine et France (récit d'une vie, témoignage recueilli par C. Lefebvre), Saint-Etienne, Starter, 2008, p. 12.

77. Dans "Nationality and the Politics of the 'Mother Tongue'», Naoki Sakai souligne le rôle des pratiques discursives dans le processus de sélection de «réalités " identifiées comme appartenant à la culture nationale, op. cit., p. 13.

78. 《住在中法大學校中的都是中國學生, 大家在一起, 說中國話, 吃中國飯, 過中國方式的 生活。》, Chen, op. cit., pp. 417-418.

79. 《我們抵達法蘭西這個文明先進之邦, 終日混在中國人海裡, 說的聽的是中國話, 吃的是 中國飯菜, 住的又像是中國房子, 起居習慣無一不中式化, 就像仍在國内一般。。。”, $\mathrm{Su}$ Xuelin, Zizhuan自傳[Autobiographie], Jiangsu wenyi chubanshe, 1996, pp. 51-52; cité et traduit par Jacqueline Estran dans «Su Xuelin et la première vague d'étudiantes à l'IFCL : Cartographie d'un désir d'ailleurs ", Transtext(e)s-Transcultures: Journal of Global Cultural Studies, Volume 9, 2014. 80. « des lieux réels, des lieux effectifs, des lieux qui ont dessinés dans l'institution même de la société, et qui sont des sortes de contre-emplacements, sortes d'utopies effectivement réalisées dans lesquelles les emplacements réels, tous les autres emplacements réels que l'on peut trouver à l'intérieur de la culture sont à la fois représentés, contestés et inversés, des sortes de lieux qui 
sont hors de tous les lieux, bien que pourtant ils soient effectivement localisables. », Michel Foucault, Dits et écrits IV : 1980-1988, Paris, Editions Gallimard, 1994, texte n³60. Une première publication de cette conférence de mars 1967 est parue sous le titre « Des espaces autres » dans Architectures, Mouvements, Continuité, n5, octobre 1984, pp.46-49.

\section{RÉSUMÉS}

L'environnement scientifique et culturelle de cette école de préparation aux études supérieures en France avait pour finalité principale de former cette diaspora intellectuelle temporaire afin qu'elle joue ensuite son rôle historique dans le devenir moderne de la Chine. Le label d'école de la modernité est donc plus que légitime. Par contre, affirmer que l'Institut puisse avoir été un lieu où des individus se sont constitués en tant que « sujets chinois » constitue une proposition moins évidente. Si l'expérience particulière de l'IFCL n'épuise pas le processus, nous faisons l'hypothèse qu'elle cristallise néanmoins des pratiques discursives qui ont contribué à façonner un «sujet chinois moderne», expression que le présent essai devra tenter d'éclairer. 